\title{
A Combined Approach for Production Parameter Selection and On-site Energy Supply Management in Manufacturing Industry
}

\author{
Pouya Ghadimi ${ }^{1}$, Seyed Smaeil Mousavi ${ }^{1}$, Wen $\mathrm{Li}^{1}$, Sami Kara ${ }^{1}$ \& Bernard Kornfeld ${ }^{1}$ \\ ${ }^{1}$ Sustainable Manufacturing and Life Cycle Engineering Research Group. School of Mechanical and \\ Manufacturing Engineering, University of New South Wales, Sydney, Australia \\ Correspondence: Pouya Ghadimi, School of Mechanical and Manufacturing Engineering, University of New \\ South Wales, Sydney 2055, Australia. Tel: 6-144-987-7892. E-mail: Pouya.ghadimi@gmail.com
}

Received: January 20, 2016

doi:10.5539/mas.v10n8p230
Accepted: February 22, 2016

Online Published: June 22, 2016

URL: http://dx.doi.org/10.5539/mas.v10n8p230

\begin{abstract}
Integrated management of manufacturing plant's production and on-site energy supply systems has shown potential economic, environmental and resource efficiency advantages for the industry. However, existing approaches are solely based on pure mathematical models with a high degree of abstraction with limited applicability, which becomes impractical for industrial applications. In this paper a simulation methodology for production parameters selection and on-site energy supply management is presented. In this case, state-based models and operational strategies of manufacturing processes and on-site energy supply options are integrated to represent interdependency between production processes, technical building services and on-site energy supply system. As a result, the proposed methodology covers manufacturing system complexity without compromising the required accuracy. This is applied to a batch based manufacturing plant and the impact of particular production parameters on energy demand profile is evaluated. The results indicate the impact of production parameters on energy supply system. In addition, the proposed approach enables manufacturers to evaluate the implications of potential production approaches in order to select appropriate operational strategies for on-site energy supply systems.
\end{abstract}

Keywords: manufacturing industry, on-site energy supply, production parameters selection

\section{Introduction}

Combined management of production and on-site energy supply systems aims to minimize energy charges, environmental impact and primary energy resource consumption of individual manufacturing plants while maintaining their competitiveness. The motivations for such an approach are threefold. Firstly, the manufacturing industry, comparing to other sectors, is one of the major consumers of energy resources with a high level of emissions associated with global warming. The report on worldwide trends in energy use and efficiency revealed that the industry not only consumed the largest share of total energy generated in 2012 (42.3\%), it was also responsible for the largest share of carbon dioxide emissions (39.3\%) (International Energy Agency, 2014). A similar trend has been observed in Australia where manufacturing is identified as the most energy intensive sector, accounting for $38.3 \%$ of the national level energy consumption (Australian Bureau of Statistics, 2014). Secondly, the consumption of material and natural resources in the manufacturing industry is increasing continuously in response to growing demand for consumer goods (GEA Writing Team, 2012). Consequently, manufacturers are seeking alternative solutions to improve their plants' efficiency and reduce associated impacts with their energy demand growth. Finally, industrial consumers are exposed to drawbacks associated with energy supply outsourcing such as high instability and rising of energy prices. Hence, the role of energy in the manufacturing industry has been changed significantly (European Commission, 2009; Union européenne, Direction générale de la recherche, 2013). Accordingly all the future solutions should inherently be more sustainable by offering methodologies to reduce energy consumption or switching to more efficient, cleaner and cheaper energy supply options.

In this context, on-site energy supply not only provides enormous economic, environmental and resource efficiency benefits but also supply of more reliable electricity and heat (Meckler \& Hyman, 2010). There is a range of available on-site energy supply options such as gas turbines, reciprocating engines and photovoltaic systems. Among these, controllable Combined Heat and Power (CHP) systems can reduce primary resource 
consumption by up to $30 \%$ compared to conventional energy supply options, such as grid interconnection that mainly rely on coal power plants with lower efficiency and high levels of transmission and distribution losses (Ruangpattana, Klabjan, Arinez \& Biller, 2011). This reduction in energy consumption is achieved through specifically tailored and simultaneous heat and power generation at the point of consumption. On-site energy supply unit can be considered as part of Technical Building Service (TBS) which covers other utility requirements such as conditioned air and Compressed Air (CA). Figure 1 presents a schematic of potential on-site energy supply in a manufacturing system.

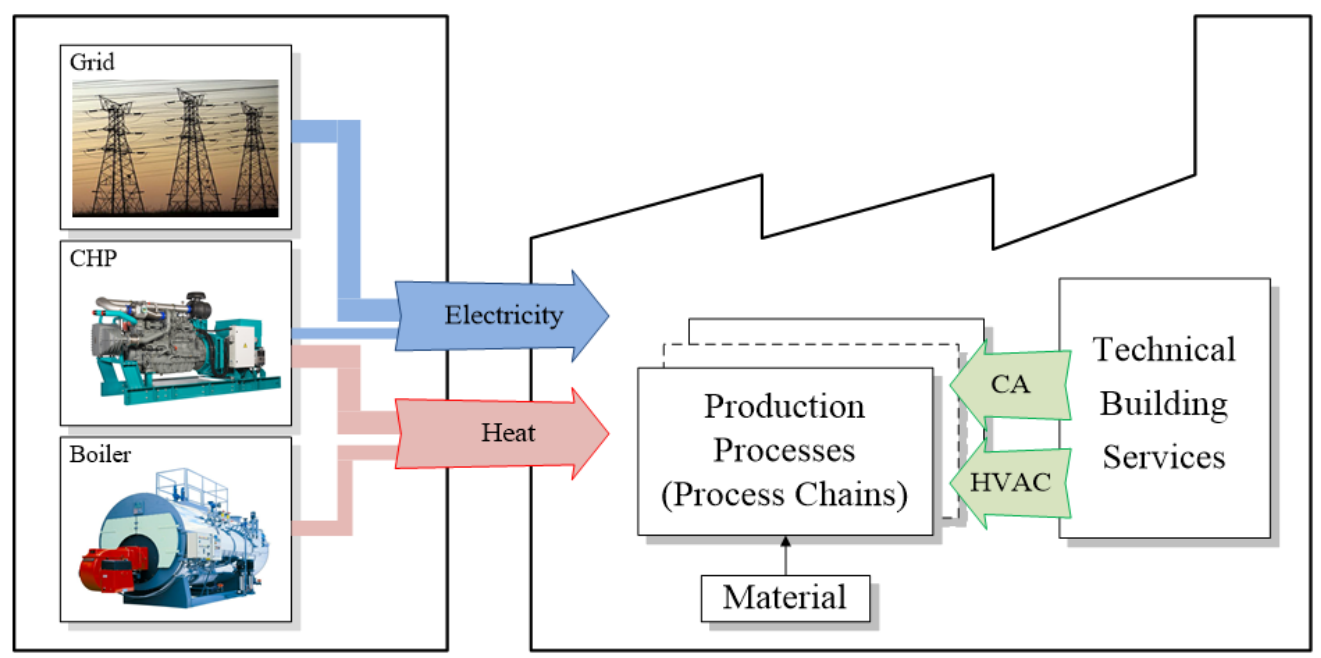

Figure 1. Schematic of on-site energy supply for manufacturing systems

Moreover, recent studies have illustrated additional system improvements can be achieved through combining production and on-site energy supply management (Agha, Thery, Hetreux, Hait \& Le Lann, 2010; Meckler \& Hyman, 2010). In these cases, production objectives such as make-span or inventory minimization are combined with energy oriented objectives such as operational cost minimization. However, the impact of production parameters on the energy demand profile and on-site energy supply system has not been studied. This should cover the interaction between the production environment and the on-site energy supply system to manage energy supply options through more efficient production parameters selection.

Consequently, the main goal of this paper is to develop a practical solution for combined management of production and on-site energy supply in manufacturing industry. The remainder of this paper is organized as follow. In Section 2, we provide an overview of related literature where the shortcomings of existing solutions are identified. Section 3 explains applied modelling and methodology to address those shortcomings. Section 4 presents the case study in which the methodology is applied to an existing batch based production system. Finally, in Section 5 we conclude the paper and propose directions for future work.

\section{Background}

\subsection{Production and Energy Interrelation}

For the purpose of this paper, machines, material and energy flows are considered as the key elements in manufacturing systems. The interrelation between these elements needs to be taken into account for combined management of production and on-site energy supply operation. In this case, energy flows associated with manufacturing system operation is directly influenced by selected production parameters. For example, varying production rate of machine tools can have a significant impact on material flow through the factory, which directly impacts energy demand profiles at process level (Mousavi, Thiede, Li, Kara \& Herrmann, 2015). A balanced material flow can reduce the energy consumption of a machine by minimising the time spent in non-productive operations such as idle mode (Pechmann, Scholer \& Hackmann, 2012). A balanced material flow can also be achieved by selecting smaller batch sizes for the products, where there are alternative machines for performing a task on a particular product in a job shop system (Stadtler, 2005). This would maximise machine utilization and reduce energy demand by the machines.

In return, the associated energy demand profiles of a manufacturing plant would affect operation of the on-site 
energy supply options (Ashok \& Banerjee, 2003). In this case, power and heat demand fluctuations determine the required output from individual units of an energy supply system to satisfy the energy balance. Moreover, in order to achieve optimal operation of the manufacturing system, a matrix of objectives such as cost, environmental impact or primary resource consumption should be taken into account. In the case of CHP operation, initial model developed by Mitra et al considered objectives including Time of Use (TOU) tariffs, associated $\mathrm{CO}_{2-\mathrm{e}}$ rates, and the results show that dynamic energy demand profiles have a significant impact. (Mitra, Sun \& Grossmann, 2013). However, the production management has been excluded in these studies.

All in all, a variable energy profile as a result of selected production parameters leads to dynamic operation and different performance from both production and on-site energy supply system perspectives. The following section reviews existing approaches in the literature that aim to address combined approach for management of production and on-site energy supply in manufacturing industry.

\subsection{Current Approaches}

Combining production and on-site energy supply planning has been identified as an important strategy to reduce energy charges and consumption. This is generally classified into sequential (Mařík, Schindler \& Stluka, 2008) and integrated (Agha et al., 2010; Mitra et al., 2013; Zhao, Rong, Feng \& Dong, 2014) solutions. The sequential approaches have the master and slave form in which manufacturing task scheduling, energy demand forecast and energy supply operation planning are carried out consecutively. On the other hand, integrated approaches couple the scheduling of manufacturing tasks with operational planning of on-site energy supply system. Although both approaches illustrate potential benefits and improvements by reducing energy charges and associated emissions, three core obstacles currently limit their application.

The first shortcoming is exclusion of some TBS components. In the existing studies, a manufacturing system is comprised of only two units, manufacturing and utility (Agha et al., 2010). Manufacturing unit covers production facilities and processing equipment representing material transformation into the final product. Similarly, the utility unit is considered as only steam, heat and electricity providers such as boilers, grid interconnection and CHP systems. However, a manufacturing plant's operation also relies on other utility providers (Figure 1). This consists of facilities such as HVAC and air compressors which provide required production environment conditions namely heating, refrigeration, conditioned air and process-related utilities such as CA (Mousavi et al., 2015). These facilities consume additional energy inputs which can contribute to a significant share of energy consumption in a manufacturing plant. Existing studies in this case illustrate that energy consumption of TBS can account for approximately $35-40 \%$ of the total energy consumption (Herrmann, Thiede, Kara \& Hesselbach, 2011). Consequently, it is necessary to bring all the TBS components into the combined studies of production and on-site energy supply management to achieve more realistic representation of energy flows in a manufacturing plant.

Secondly, both manufacturing and utility units are mathematically modelled to cover problem objectives, input-output correlation and generic system constraints (Agha et al., 2010). In this case, resource task networks are used to represent materials, processing, storage and transformation carried out in a manufacturing unit. The authors used an energy oriented input-output model is used to represent the utility unit. Although these cover generic constraints such as material balance, capacity, shutdowns and restarts, they are limited in comprehensive representation of industrial systems due to their assumptions and simplifications. Illustration of operation planning is a time-based problem that requires integration of the machines and processes operational states. Existing mathematical modelling approaches do not take into account existing modes of operation associated with individual units. The importance of operational states in representing the transient performance of production processes and energy requirement has been illustrated in several studies (Mouzon \& Yildirim, 2008; Mouzon, Yildirim \& Twomey, 2007). In this case, comprehensive models should be implemented to take into account operational states of individual units such as changeover, stand by, start up and cooling down (P. Ghadimi, Kara \& Kornfeld, 2014; Herrmann et al., 2011; Mitra et al., 2013; Mousavi et al., 2015).

Thirdly, regardless of significant progress in mathematical modelling, industrial problems are still challenging to be solved due to the inherent complexity associated with system representation and interdependencies in batch based production systems. The difficulty associated with implementation of an integrated production and utility optimization methodology is reported as an existing limitation for industrial applications due to intensive computational requirement with slower convergence times (Agha et al., 2010; Sun, Ding \& Li, 2012).

In summary, existing approaches lack of practicality owing to the exclusion of TBS component as well as over-simplification of the complex production and on-site energy generation systems. Consequently, this study tackles a practical approach for combining production and on-site energy supply management within the 
identified boundaries. The following section describes the proposed modelling approach to address these shortcomings.

\section{Modelling Approach}

In the proposed combined production and on-site energy supply framework, a hierarchical factory structure is considered and a model of the factory containing two modules-namely production processes and TBS are integrated as shown in Figure 2. In this case, the production processes module is modelled to estimate energy carrier requirements. Following this, the TBS module estimates the total energy demand profile of the factory and these profiles are iteratively passed to the on-site energy supply unit to simulate potential operational strategies of energy supply options and calculate energy related objectives. The simulation approach is used in this study to overcome the limitations explained in section 2 (Khoo, Spedding, Tobin \& Taplin, 2001). The following sections explain details associated with individual modules and their integration.

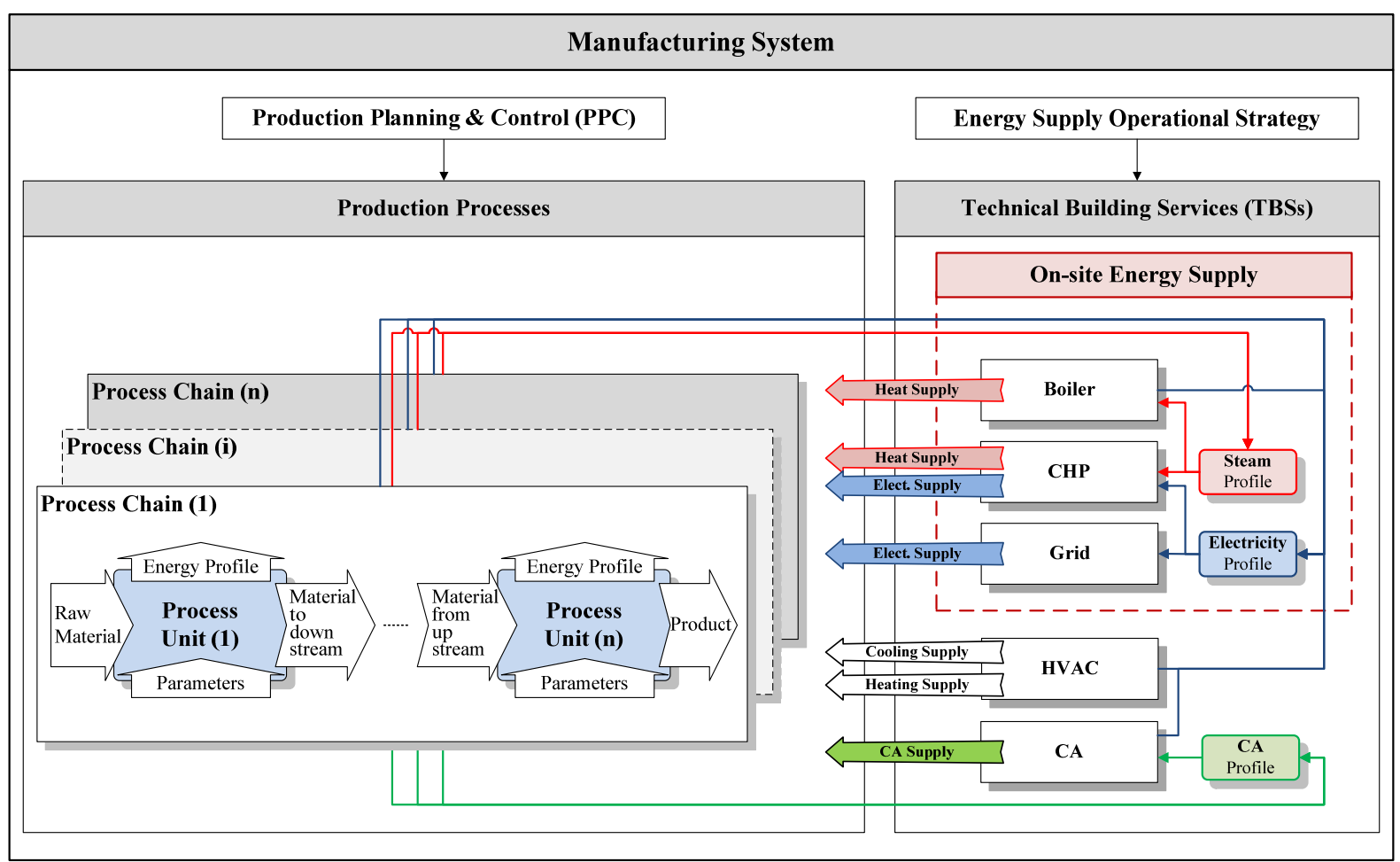

Figure 2. Combined production and on-site energy supply framework

\subsection{Production Processes Module}

The production processes module covers physical structures of the manufacturing system, existing constraints and technical requirements as well as the flow of material, and energy carriers. A state-based modelling technique was used to develop this module in the previous work of authors (Mousavi et al., 2015). This module considers production plan and parameters to estimate energy carrier requirements including electricity, heat/steam and CA.

In this framework, the production processes consist of one or several process chains in which each is developed by selecting one process unit for each machine tool (Figure 2). This unit forms the basic element in the framework towards predicting energy demand (Mousavi et al., 2015). Later on, individual process units are connected to each other by considering process layout and operational sequences. Finally, the production processes module receives a production schedule from Production Planning \& Control (PPC) as input and simulate the flow of material. Each process unit is configured separately through parameters' set in the PPC. As a result, energy profiles of individual process units are determined which in turn estimates energy carrier requirements.

More specifically, the PPC is where some typical production parameters such as machine settings or batch sizing can be determined. Machine settings have shown significant impact on energy consumption at both process unit 
(Kara \& Li, 2011) and process chain level (Mousavi et al., 2015). For example, production rate of bottleneck machine(s) is a critical parameter which may change work load on the machine as well as throughput of the system. Batch sizing is another parameter, which can be considered. Although selecting smaller batch size increases number of changeovers and non-productive times, it can result in a more balanced work load and avoid stocking product before machines.

\subsection{TBSs Module}

Production processes consume utilities such as CA, steam/heat and conditioned air that are mainly provided by TBSs (Herrmann et al., 2011). These utilities are usually a function of operations in production processes and their supply results in additional energy requirement, which should be added to the production processes energy demand profile. Five units were modelled in this framework namely, Boiler, CHP, Grid, HVAC and CA. The details of the validated models for Boiler, CHP and CA units can be found in previous work of authors (P. Ghadimi et al., 2014; Mousavi et al., 2015). Depending on the requirement of these utilities in the system, the related units can be added to the module. Associated parameters for individual units are set in the PPC.

As an example, the CA unit operates based on the compressed air demand of production processes simulated as part of the production processes module, which in turn estimates CA unit's electricity demand profile. This profile is then added to factory's energy demand and eventually the total demand of the factory for electricity is determined.

\subsubsection{On-Site Energy Supply}

As illustrated in Figure 2, the on-site energy supply unit is integrated as part of the TBS to represent operation of energy supply options subject to potential operational strategies. The unit receives the simulated energy demand profiles over the specified time horizon and determines its components' operational signals based on the selected operational strategy.

In order to achieve this, state-based models of individual energy supply units are used. Basic models have been developed and verified in the previous work of the authors (P. Ghadimi et al., 2014). These cover underlying input-output models to enable a comprehensive coverage of operational states, such as start-up, idle, cooling down, and block states in addition to associated transitions and operational constraints for individual states.

Moreover, two classes of operational strategy are modelled. The first class is load-following, in which the energy supply components follow the signalled energy demand in a time-series format regardless of associated charges, primary resource consumption share and availability of other options. This represents a rule-based control strategy, which is the most commonly used operational strategy, due to its simplicity and independent communication requirements. In addition, to increase the potential outcome of an on-site energy supply system an optimal operational strategy is developed. This should comprehensively take into account desired objective functions and operational constraints which are modelled through optimization programming (Pouya Ghadimi, Kara \& Kornfeld, 2015). The aforementioned approach forms a Model Predictive Control (MPC) strategy which takes into account the operational constraints of individual components such as upper and lower bounds of operational loads, ramp rate constraints in addition to energy system constraints such as required energy balance. These strategies cover energy tariffs (for both electricity and gas) based on the factory's existing contract with energy suppliers such as Time of Use (TOU) tariff for electricity purchase. Moreover, intelligent start-up scheduling of the CHP systems is included. Consequently, this can generate optimal dispatch signals for individual units with respect to their associated operational cost, environmental impact and primary resource consumption defined as the objective function.

\subsection{Modules Interdependency}

Ultimately, integration of the aforementioned modules is carried out in the simulation environment. In general, there are three steps which need to be followed from case to case.

As the first step, model setting is performed which includes the selection of decision variables and performance indicators as well as design of simulation scenarios. To be more specific, the selection of decision variables is dependent on the flexibility of the studied case. Often, expert opinion is taken into account when select those variables. This can result in a big and complex solution space to optimize the system. To handle this, there are several generic methods such as Design of Experiment (DoE) to narrow down the study and focus on only a small part of the solution space to make general interpretation. In particular, factional factorial design is an effective approach when multiple decision variables are selected. The number of variance level for each decision variables need to consider the system complexity and associated industrial constraints. Thus, the number of total scenarios is always case specific. 
The selection of performance indicators are aligned with the objective of the integration and optimisation. Typical performance indicators can be classified into economic indicators (e.g., cost) and environmental indicators (e.g., energy associated carbon footprint).

After model setting, each simulation scenario is used to configure the combined model. According to the DoE, different energy demand profiles are generated by use of the production process module and utilised in the TBS module. Simultaneously, the on-site energy module is simulating the production of on-site energy against different operation strategies such as load following, or optimised, etc. These bring in production processes, TBS modules and on-site energy supply unit together in an advanced manner by taking into account system complexity and associated constraints.

Finally, the objective values are calculated according to the selected performance indicators for each simulation scenarios. For the case of single objective optimisation, the optimal scenario can be easily obtained, whereas the trade-off among multiple objectives can be also analysed. In the latter case, a weighted max-min approach can be used to determine the optimal setting. In addition, further investigation can be performed to determine the significant process parameters/decision variables which has an impact on the overall system performance. Moreover, different operation strategies of on-site energy system can be evaluated concurrently.

\section{Case Study}

The proposed approach was applied to a batch-based pharmaceutical manufacturing system in Australia. The company follows a make-to-stock production system to produce sterile injectable solutions. Figure 3 illustrates the system boundaries of the case study.

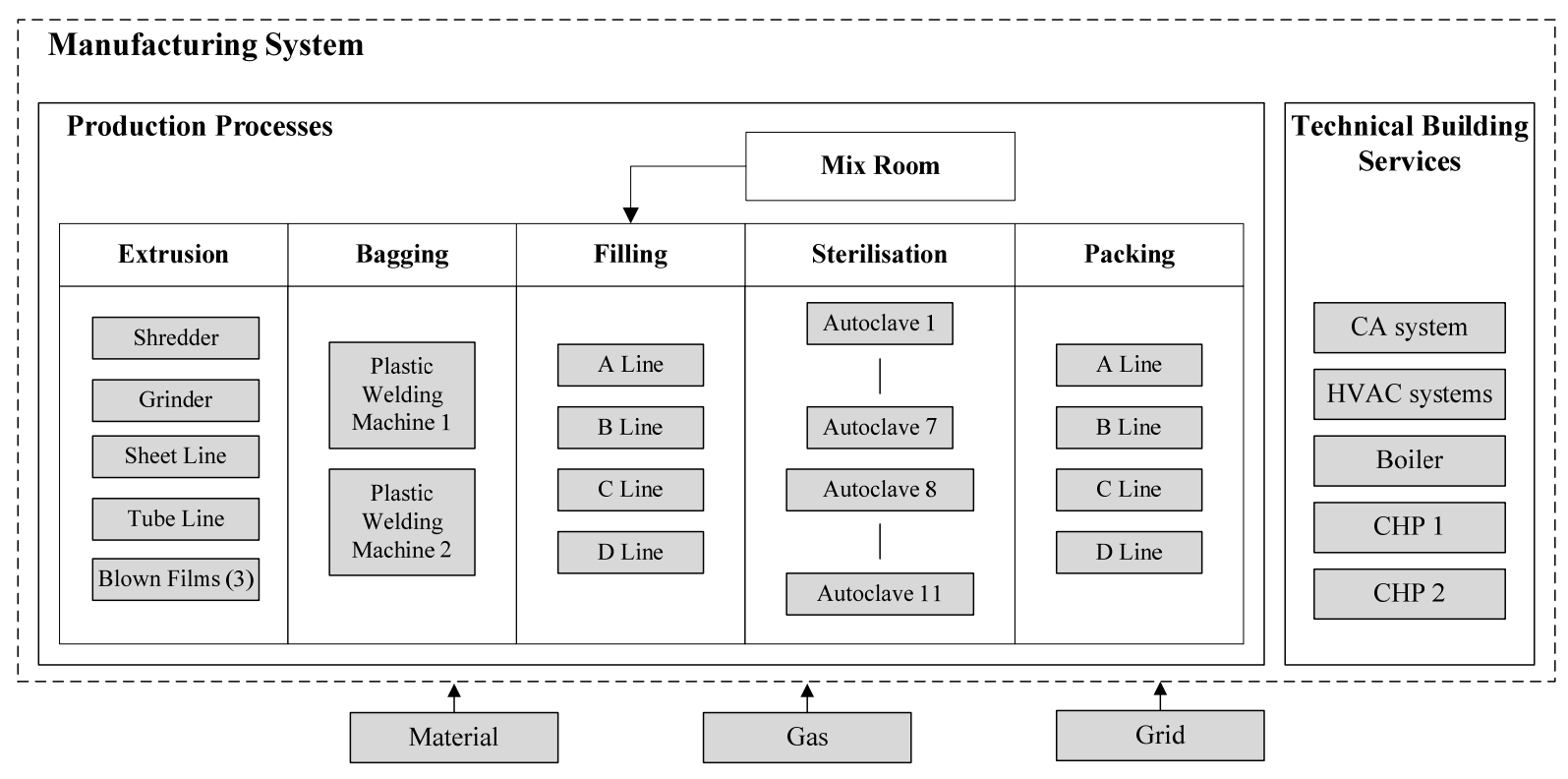

Figure 3. System boundaries of the selected case study

As illustrated in Figure 3, production follows the production schedule and commences by pulling raw plastics from storage and converting them into plastic rolls through the extrusion line. Later on these rolls are transferred to the two plastic welding machines to manufacture solution bags through the bagging line. A mixing process that includes filtration, reverse osmosis, chlorination, and distillation is operated concurrently to produce water, which is then stored in buffer tanks to prepare processed solution. This is later on filled into the bags through four filling lines. Then, the products are collected into part-batches and transferred to autoclaves to complete the sterilisation process. Finally the sterilised products are transferred to the packing area and the warehouse.

An on-site energy supply system provides the required electricity and heat demand of the whole factory through existing transformers and heat distribution system. This system consists of grid interconnection, boiler and reciprocating engine based CHP systems in addition to HVAC and compressed air system (Figure 3). In this case, individual processes and machineries are connected to specific transformers in which electricity demand of each 
transformer is supplied by one CHP system and grid interconnection.

The following sections review application of the model to generate combined production and on-site energy supply management scenarios. The case study aims to evaluate and find the production parameter settings of bottleneck processes to achieve cost-effective operation of on-site energy generation. Following this, simulated system performance indicators are presented and evaluated for individual scenarios.

\subsection{Model Setting}

The proposed methodology was applied and the case was modelled in Anylogic simulation package 6.9. In this case, firstly the critical decision variables of the production process module were identified (section 4.1.1). Following this a set of scenarios were generated to replicate integration of the two modules (section 4.1.2). Finally, performance indicators were selected and the manufacturing system was simulated subject to potential operational strategies of the on-site energy supply (section 4.1.3). The achieved results were also compared with the system operation in absence of the CHP system.

\subsubsection{Decision Variables}

To generate potential production planning scenarios, several decision variables were considered and process flexibility with respect to them were investigated subject to industrial partner approval. In this case, filling lines were known as bottleneck and their production capacity was constrained. Therefore increasing the production rates by reducing filling line cycle times was considered as a key factor for improvement.

Based on the collected historical data, $\pm 10 \%$ variation of the filling lines' production rate were set. As the outputs of the filling lines use shared sterilisation machines, a reduction in one line's production rate can increase the sterilisation capacity to be used for other lines' output. For example, a 10\% increase in production rate of a particular line reduces the cycle time by the same rate. In terms of energy consumption, it was assumed that there is linear relationship between power rate of the machine during production and production rate $(\mathrm{Li}, 2015)$. Furthermore, the production plan is set to produce the same amount of product in each shift.

\subsubsection{Scenario Design}

To study the impact of selected variables on the performance of the system, a central composite design was selected as the case includes continuous parameters in three levels. In this case, a face centred design was used as presented in Table 1. Particularly a fractional factorial design including 25 points defined potential scenarios. The current status of the systems, which is known as baseline scenario (BL), is defined by setting all the investigated variables to zero. For each scenario, the model was set to run for three weeks, with one week dedicated as the transient phase to exclude any influence from the initial conditions.

Analysis of on-site energy supply for the case study was carried out based on the simulated electricity and heat demand profiles and subject to the two main control strategies described in section 3.2.1.

Table 1. List of considered variables and their boundaries

\begin{tabular}{lllll}
\hline Machine & Variable & Lower Bound & Current Value & Upper Bound \\
\hline illing line A & & $-10 \%$ & 0 & $+10 \%$ \\
Filling line B & Production rate & $-10 \%$ & 0 & $+10 \%$ \\
Filling line C & $-10 \%$ & 0 & $+10 \%$ \\
Filling line D & & $-10 \%$ & 0 & $+10 \%$ \\
\hline
\end{tabular}

\subsubsection{Performance Indicators}

Four Key Performance Indicators (KPIs) were considered to evaluate defined scenarios. Throughput, as the first indicator, was used to quantify the total production volume of the system in a given time period. The second KPI was Total Primary Energy (TPE) to specify total energy consumption by the entire manufacturing system including electricity purchased from grid and gas consumption by the boiler and two CHP systems in addition to associated losses. Moreover, Total Energy Cost (TEC), as the third KPI, determined the monetary value of TPE consumption. Finally, Total Environmental Impact (TEI) was measured to indicate $\mathrm{CO}_{2 \text {-e }}$ footprint of the system's operation covering both electricity and gas consumption.

The aforementioned approach enabled the assessment of potential combined operation plans of production and on-site energy supply system. The following sections present results associated with individual simulation scenarios. 


\subsection{Production Parameters and Energy Demand Profile}

The key production parameter impact on the energy demand profiles was investigated through this case study. In this case, the proposed approach covered both electricity and heat demand fluctuations in a timely manner with respect to production rate.

Figure 4 illustrates the impact of filling lines' production rate selection compared with the BL scenario representing current production plan for one day. As it is shown, maximising production rates up to $10 \%$ for all the filling lines as scenario 16 (S16) have direct impact on both electricity and heat demand levels and its timing compared to the BL.

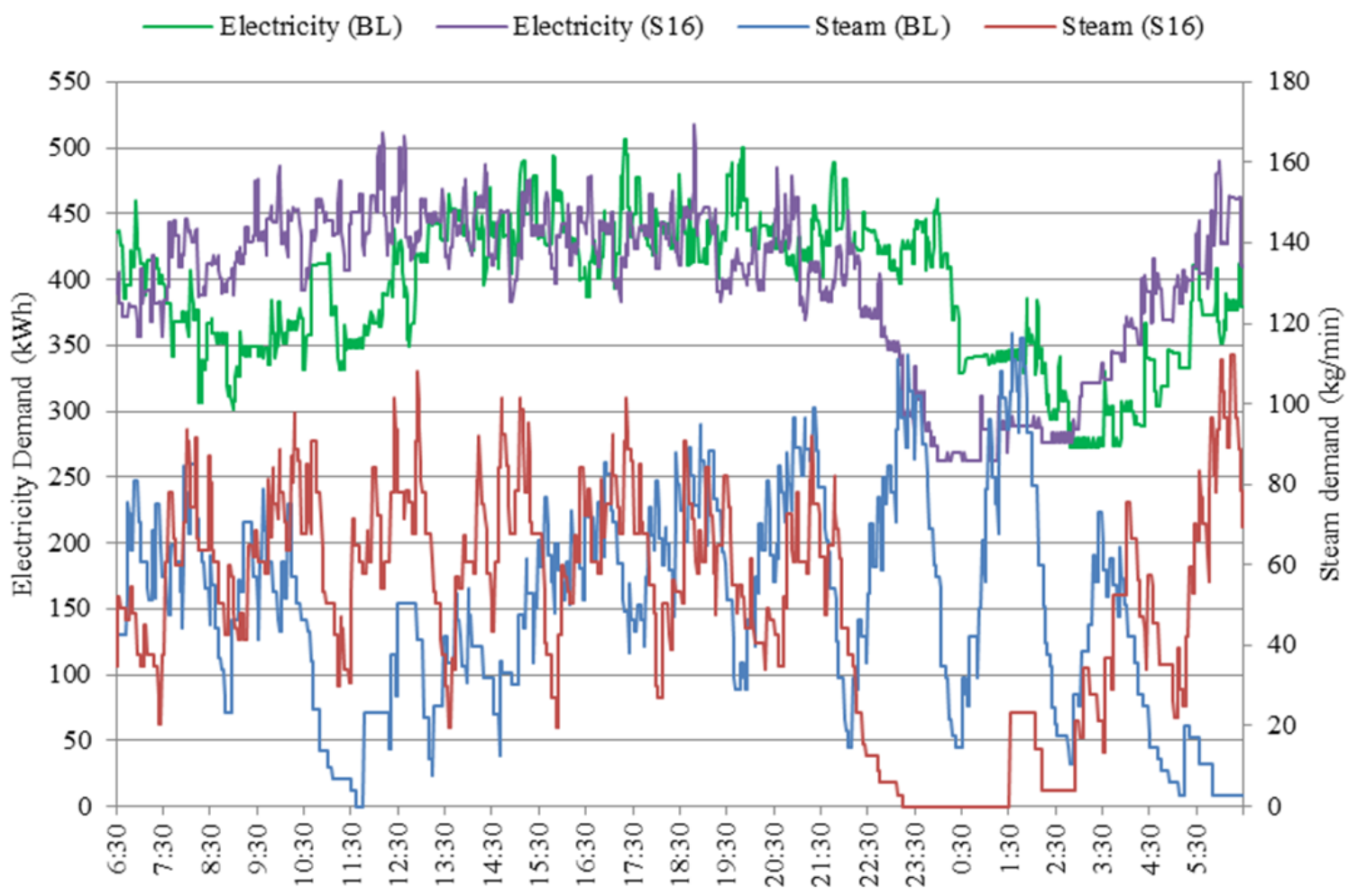

Figure 4. Impact of production rate selection on electricity and steam demand (one day sample)

In this case, higher production rates subject to S16 result in increased throughput at the start of the day, shifting electricity demand to earlier time in the morning compared to BL scenario. This impact can be seen for both electricity and steam demand profiles. The next sections review the impact of this change on the operation of on-site energy supply options subject to potential operational strategies.

\subsection{Load Following Operational Strategy}

In this scenario, the designed production plans were simulated in presence of the on-site energy supply, and subject to a load following operational strategy (LFS). Figure 5 illustrates impact of the edited energy demand profile as a result of production rate selection on the CHP 2 operation. The latter is compared with the CHP operation subject to the BL energy demand profile. 


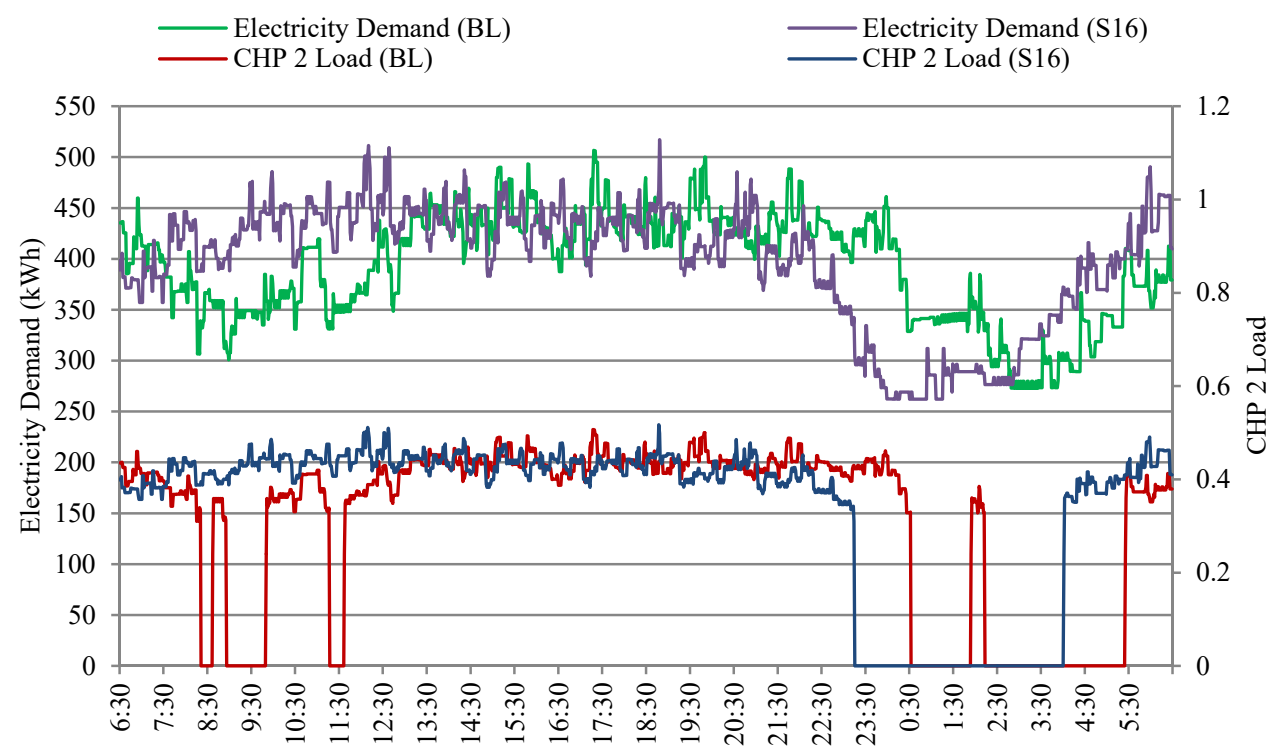

Figure 5. Electricity demand and CHP load correlation subject to LFS (one day sample)

In this case, the S16 illustrated improved system performance compared to the BL scenario. As it is shown, a more consistent operation of CHP system is achieved for S16 (CHP2 Load (S16)), as the energy demand level is higher in a shorter time span. Moreover, the heat demand shift to earlier hours resulted in boiler shutdown from 2:30 to 4:30 subject to S16.

\subsection{Optimal Operational Strategy}

Similar to the LFS, potential production plans were simulated to forecast energy demand trajectories. Following this the on-site energy supply strategy was simulated subject to Optimal Operational Strategy (OS). In this case, economic optimization is the core of the simulation, which takes into account the TOU tariff structure of grid purchased electricity, associated charges of individual energy supply options, their availability and operational constraints in the form of an optimization problem to minimise energy charges. As an example, Figure 6 illustrates electricity demand of one transformer for the scenario one (S1), where production rates are minimised $(-10 \%)$ for all lines. Moreover, the load on CHP 2 is presented for both LFS and OS operational strategies.

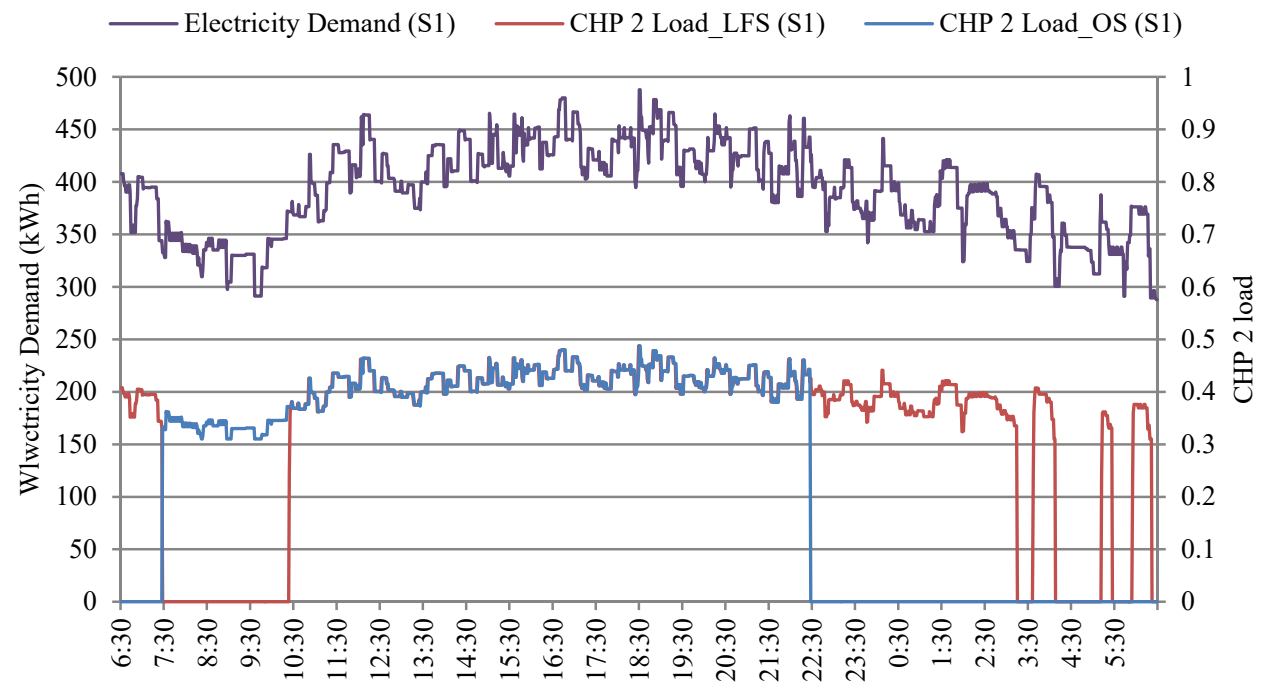

Figure 6. Electricity demand and CHP load correlation subject to OS (one day sample) 
As illustrated a more consistent operation of CHP is achieved and the impact of TOU tariff is taken into account in the OS (CHP 2 Load-OS (S1)). In this case, as the plant's operation reaches off-peak times the energy supply switches to the grid electricity purchase. This is due to economic MPC and the higher charge rate of the gas compared to off-peak purchased electricity charges.

The following section compares the system performance subject to aforementioned operational strategies and manufacturing plant operation without CHP systems.

\subsection{Operational Strategies Evaluation}

The following figures compare the share of energy cost, energy supply and environmental impact of the best scenarios with minimised KPIs values identified in different strategies; subject to three cases namely, no CHP, CHP subject to LFS and CHP subject to OS. Figure 7 demonstrates energy cost share of different energy supply options for 2 weeks under different strategies.

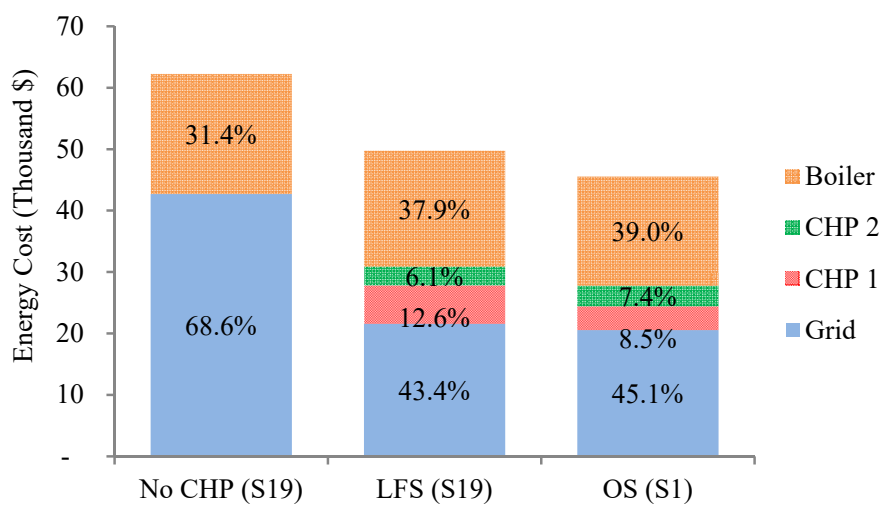

Figure 7. Energy cost share of the best scenarios for different cases

In both no CHP and LFS, scenario 19 (S19) where only production rate of filling line B is reduced by $10 \%$, resulted in minimum energy cost. However, selected production rates for $\mathrm{S} 1$ resulted in minimised values subject to OS as shown in the last column. According to Figure 7, great share of energy cost can be saved by CHP system implementation subject to both LFS $(-20.1 \%)$ or OS $(-26.8 \%)$ compared to the case where there is no CHP. Figure 8 illustrates identified scenarios for each case with minimum total energy consumption.

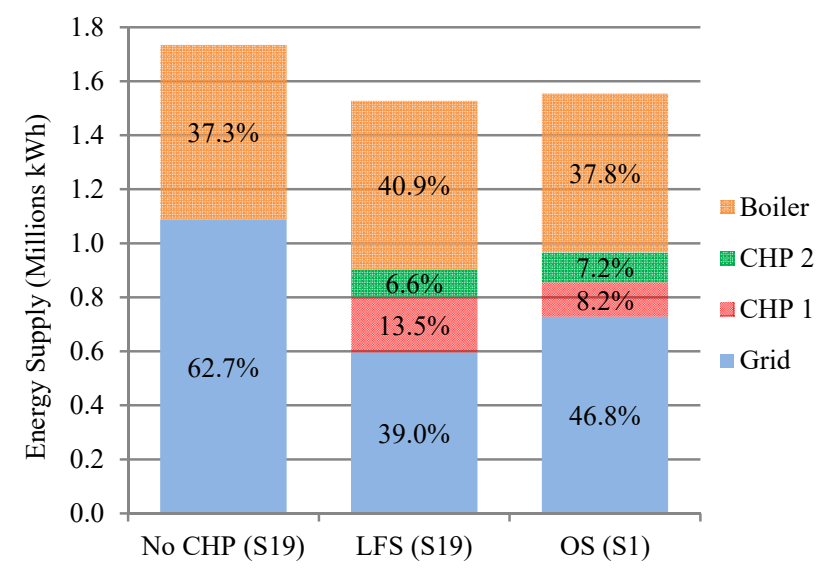

Figure 8. Energy supply share of the best scenarios for different cases

According to Figure 8, a great share of energy can be saved by CHP system implementation subject to both LFS $(-12.0 \%)$ and OS (-10.4\%) compared to the case where there is no CHP. Total energy consumption of the system in both LFS and OS are similar (1527145 and $1554791 \mathrm{kWh}$ respectively). However energy cost of the entire system in OS is around $8.5 \%$ less than LFS (see Figure 7). This is due to the economic based optimisation of the OS that selects to consume less gas and purchase more electricity from grid as electricity rate during off-peak times is less than gas charges. The higher share of TPE subject to OS is due to additional purchased grid 
electricity with lower efficiency. Figure 9 illustrates identified scenarios for each case with minimum TEI.

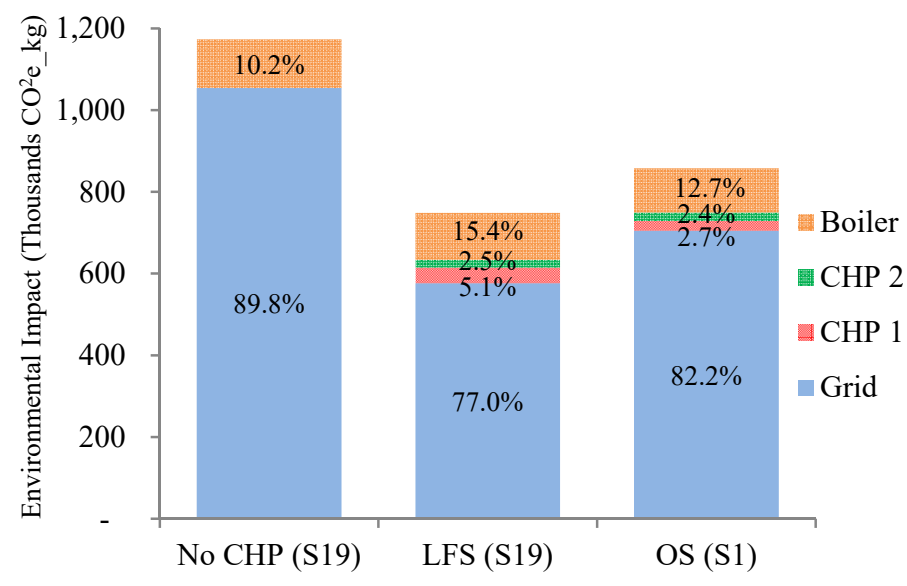

Figure 9. Environmental impact share of the best scenarios for different cases

According to Figure 9, a great share of environmental impact can be saved by CHP system implementation subject to both LFS (-36.2\%) and OS (-26.9\%) compared to the case where there is no CHP. However, the total environmental impact of the system in OS is higher than LFS (14.6\%). This is due to the fact that CHP operation subject to the OS purchase higher share of grid electricity during off-peak times with higher rate of $\mathrm{CO}_{2-\mathrm{e}}$.

\section{Conclusion}

Although existing studies illustrate potential improvements through integrated planning of production and on-site energy supply, their practicality is limited due to fundamental assumptions and restricted implementation as a result of problem complexity. This study implemented a simulation-based approach in which comprehensive models and constraints of the production environment, TBS and on-site energy supply systems are combined together. In this case, state-based models of individual machines, processes and energy supply units result in comprehensive system modelling and more realistic handling of system operation constraints.

Furthermore, the proposed approach enabled coverage of the potential production parameters selection and evaluation of their impact on the energy demand profiles and on-site energy supply system operation. As shown, both production rate and selected operational strategies affect the share of energy supplied from individual components.

In this case, the production strategies with specific production rates were identified to minimise associated values of selected KPIs subject to individual operational strategies. It should be noticed that as the optimal operational strategy is simulated in the form of an economic optimisation, the operation of the CHP systems are planned to minimise energy charges by being switched off during off-peak hours due to cheaper cost of grid electricity.

As a result, a practical solution to minimise operational costs of on-site energy supply systems with high convergence rate is achieved. The proposed approach enabled quick evaluation of the potential production approaches for management level as a decision making tool along with on-site energy supply operational strategy selections.

Further research will be conducted to consider multiple production parameters, implement multi-objective optimisation to bring in different KPIs together, as well as the value of the energy storage systems.

\section{Acknowledgment}

This project is funded by the Australian Research Council in collaboration with Baxter Healthcare Australia.

\section{References}

Agha, M. H., Thery, R., Hetreux, G., Hait, A., \& Le Lann, J. M. (2010). Integrated production and utility system approach for optimising industrial unit operations. Energy, 35(2), 611-627. http://dx.doi.org/10.1016/j.energy.2009.10.032 
Ashok, S., \& Banerjee, R. (2003). Optimal operation of industrial cogeneration for load management. Power Systems, IEEE Transactions on, 18(2), 931-937. http://dx.doi.org/10.1109/TPWRS.2003.811169

Australian Bureau of Statistics. $\quad$ (2014, $\quad$ February). http://www.abs.gov.au/ausstats/abs@.nsf/Lookup/4604.0main+features42011-12\#ENERGY\%20INTENSIT $\mathrm{Y}$

European Commission. (2009). ICT and Energy Efficiency: The Case for Manufacturing. Luxembourg: European Commission Information Society and Media.

GEA Writing Team. (2012). Global Energy Assessment: Toward a Sustainable Future. Cambridge: Cambridge University Press.

Ghadimi, P., Kara, S., \& Kornfeld, B. (2014, June 2-6). Reactive modelling of on-site energy system components for real-time application. The 2014 IEEE International Conference on Intelligent Energy and Power Systems (IEPS). http://dx.doi.org/10.1109/IEPS.2014.6874183

Ghadimi, P., Kara, S., \& Kornfeld, B. (2015). Real-Time Operation Management of CHP System in Manufacturing Industry. Modern Applied Science, 9(2), 158-174. http://dx.doi.org/10.5539/mas.v9n2p158

Herrmann, C., Thiede, S., Kara, S., \& Hesselbach, J. (2011). Energy oriented simulation of manufacturing systems-Concept and application. CIRP Annals-Manufacturing Technology, 60(1), 45-48. http://dx.doi.org/10.1016/j.cirp.2011.03.127

International Energy Agency. (2014). Key World Energy Statistics.

Kara, S., \& Li, W. (2011). Unit process energy consumption models for material removal processes. CIRP Annals-Manufacturing Technology, 60(1), 37-40. http://dx.doi.org/10.1016/j.cirp.2011.03.018

Khoo, H. H., Spedding, T. A., Tobin, L., \& Taplin, D. (2001). Integrated Simulation and Modelling Approach to Decision Making and Environmental Protection. Environment, Development and Sustainability, 3(2), 93-108. http://dx.doi.org/10.1023/a:1011616414761

Li, W. (2015). Efficiency of Manufacturing Processes (1st Ed.). Switzerland: Springer International Publishing.

Mařík, K., Schindler, Z., \& Stluka, P. (2008). Decision support tools for advanced energy management. Energy, 33(6), 858-873. http://dx.doi.org/10.1016/j.energy.2007.12.004

Meckler, M., \& Hyman, L. B. (2010). Sustainable on-site CHP systems; Design, Construction, and Operation. New York: McGraw-Hill.

Mitra, S., Sun, L., \& Grossmann, I. E. (2013). Optimal scheduling of industrial combined heat and power plants under time-sensitive electricity prices. Energy, 54, 194-211. http://dx.doi.org/10.1016/j.energy.2013.02.030

Mousavi, S., Thiede, S., Li, W., Kara, S., \& Herrmann, C. (2015). An integrated approach for improving energy efficiency of manufacturing process chains. International Journal of Sustainable Engineering. http://dx.doi.org/10.1080/19397038.2014.1001470

Mouzon, G., \& Yildirim, M. B. (2008). A framework to minimise total energy consumption and total tardiness on a single machine. International Journal of Sustainable Engineering, 1(2), 105-116. http://dx.doi.org/10.1080/19397030802257236

Mouzon, G., Yildirim, M. B., \& Twomey, J. (2007). Operational methods for minimization of energy consumption of manufacturing equipment. International Journal of Production Research, 45(18-19), 4247-4271. http://dx.doi.org/10.1080/00207540701450013

Pechmann, A., Scholer, I., \& Hackmann, R. (2012). Energy efficient and intelligent production scheduling-evaluation of a new production planning and scheduling software. The 19th CIRP International Conferencwe on Life Cycle Engineering. http://dx.doi.org/10.1007/978-3-642-29069-5_83

Ruangpattana, S., Klabjan, D., Arinez, J., \& Biller, S. (2011). Optimization of on-site renewable energy generation for industrial sites. The 2011 IEEE/PES Power Systems Conference and Exposition (PSCE). http://dx.doi.org/10.1109/PSCE.2011.5772448

Stadtler, H. (2005). Production Planning and Scheduling. In H. Stadtler \& C. Kilger (Eds.), Supply Chain Management and Advanced Planning; Concepts, Models, Software and Case Studies (pp. 199-216). New York: Springer Berlin Heidelberg. http://dx.doi.org/10.1007/978-3-540-74512-9_11

Sun, Y. M., Ding, R., \& Li, Q. Q. (2012). Research on Integrated Optimization for Production Process and Steam

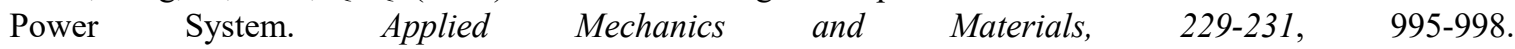


http://dx.doi.org/10.4028/www.scientific.net/AMM.229-231.995

Union européenne. Direction générale de la recherche. (2013). Factories of the Future: Multi-annual Roadmap for the Contractual PPP Under Horizon 2020. Europe: Publications office of the European Union.

Zhao, H., Rong, G., Feng, Y., \& Dong, X. (2014). Integration Optimization of Production and Utility System for Refinery-wide Planning. The 19th World Congress of the International Federation of Automatic Control, Cape Town, South Africa.

\section{Copyrights}

Copyright for this article is retained by the author(s), with first publication rights granted to the journal.

This is an open-access article distributed under the terms and conditions of the Creative Commons Attribution license (http://creativecommons.org/licenses/by/3.0/). 